\title{
Pancreatic Solid Focal Lesions: Differential Diagnosis between Autoimmune Pancreatitis and Pancreatic Cancer
}

\author{
Petr Dite ${ }^{a, b}$ Ivo Novotny ${ }^{c}$ Jana Dvorackovab, d, e Bohuslav Kianickaf,g \\ Martin Blaho ${ }^{a, b}$ Pavel Svoboda ${ }^{a, b}$ Magdalena Uvirova ${ }^{\text {e Tomas Rohan }}{ }^{\text {, }}$ h \\ Hana Maskovag, i Lumir Kunovsky ${ }^{g, i, j}$

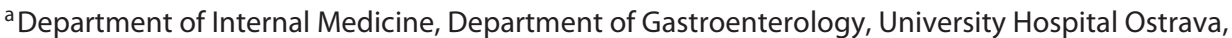 \\ Ostrava, Czech Republic; ${ }^{b}$ Faculty of Medicine, University of Ostrava, Ostrava, Czech Republic; ${ }^{\mathrm{C}}$ Department of \\ Gastroenterology, Masaryk Memorial Cancer Institute, Brno, Czech Republic; ${ }^{\mathrm{d}}$ Department of Pathology, University

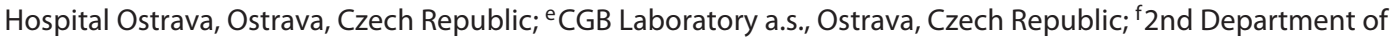 \\ Internal Medicine, Department of Gastroenterology, St. Anne's University Hospital, Brno, Czech Republic; ${ }^{9}$ Faculty of \\ Medicine, Masaryk University, Brno, Czech Republic; ${ }^{\text {h}}$ Department of Radiology, University Hospital Brno Bohunice, \\ Brno, Czech Republic; 'Department of Gastroenterology and Internal Medicine, University Hospital Brno Bohunice, \\ Brno, Czech Republic; jDepartment of Surgery, University Hospital Brno Bohunice, Brno, Czech Republic
}

\section{Keywords}

Chronic pancreatitis - Autoimmune pancreatitis · Pancreatic adenocarcinoma Paraduodenal pancreatitis $\cdot$ Pancreatic tumor $\cdot$ Immunoglobulin G4

\begin{abstract}
Background: Diagnosis of pancreatic cancer (PC) in early stages is still challenging for gastroenterologists. The early detection of cancer is one of the utmost importance for the successful therapy of this malignancy. An accurate differential diagnosis of focal pancreatic lesions plays also an important role, whether it is differential diagnosis of chronic pancreatitis from PC or autoimmune pancreatitis (AIP) from PC. Raised serum immunoglobulin G4 (lgG4) levels to twice the normal value are considered one of significant diagnostic features of type 1 AIP. However, IgG4 can be increased also in patients with $\mathrm{PC}$, but levels usually do not exceed twice the normal value. Methods: In years 2012-2017, lgG4 serum lev-
\end{abstract}

els were examined in 115 patients with histologically confirmed PC. Patients with PC and elevated IgG4 level (above $135 \mathrm{mg} / \mathrm{dL}$ ) had tested their histological resection specimens or bioptic specimens from pancreatic lesion, with targeted detection of the presence of IgG4 and plasmocytes in the pancreatic tissue and changes characteristic for type 1 AIP. Results: A plasmatic lgG4 level in 115 patients with diagnosed PC was higher than $135 \mathrm{mg} / \mathrm{dL}$ in 14 patients (12.2\%). Out of them, 2 patients (1.7\%) revealed a serum IgG4 level higher than double the normal value, that is, higher than $270.0 \mathrm{mg} / \mathrm{dL}$ (suggestive of AIP). One patient met histological criteria for diagnosis of AIP in the simultaneous presence of PC. Conclusion: Diagnosis of early cancer stages, particularly differentiating AIP from PC can be sometimes problematic. IgG4 levels can be slightly elevated also in case of PC. A targeted biopsy of the pancreas is the method of choice in cases suspected from a focal form of AIP and we recommend to prefer it over other modalities, such as, for example, response to steroid therapy.

\section{KARGER}

(c) 2019 S. Karger AG, Basel

E-Mail karger@karger.com

www.karger.com/ddi
Lumir Kunovsky, MD, PhD

Department of Gastroenterology and Internal Medicine

University Hospital Brno Bohunice, Faculty of Medicine, Masaryk University

Jihlavska 20, CZ-62500 Brno (Czech Republic)

E-Mail lumir.kunovsky@gmail.com 


\section{Introduction}

Pancreatic focal lesions can be divided into solid, fluid (cystic), and combined. Therapeutic response in solid pancreatic lesions is proportional to the accurate identification of their etiology. Differential diagnosis of malignant pancreatic conditions from chronic pancreatitis and autoimmune pancreatitis (AIP) is deemed crucial in this process. Although AIP is an uncommon disorder, its diagnosis and especially differential diagnosis can be often very challenging. Both conditions may initially manifest with similar clinical symptoms, such as jaundice in the absence of abdominal pain, for which patients are seeking medical attention. Similarly, a solid mass found predominantly at the pancreatic head region is a characteristic sign of both focal form of AIP and pancreatic cancer (PC) [1]. Imaging findings of both pancreatic tumor of the head of the pancreas and focal form of AIP may be suggestive of paraduodenal pancreatitis [2], formerly known as "groove pancreatitis" [3] or "cystic dystrophy of heterotopic pancreas" [4].

Paraduodenal pancreatitis is most commonly diagnosed in individuals aged 40-50 years and mainly in males, which is untypical either for PC or for AIP, since these conditions are clinically manifested usually in older age [5]. In females, paraduodenal pancreatitis is detected in $10-20 \%$ of all cases and similarly, $10-20 \%$ of cases of paraduodenal pancreatitis are reported in non-alcoholics. Alcohol consumption and the presence of ectopic pancreatitis tissue in the duodenal wall are believed to be key factors in the development of paraduodenal pancreatitis [6]. Increasingly viscous pancreatic secretions, typically found in individuals with a history of alcohol abuse, predisposition to calculi formation and higher concentration of the protein in pancreatic secretions all play an important role in the pathogenesis of paraduodenal pancreatitis. Brunner's gland hyperplasia, also linked to alcohol consumption, leads to obstruction or dysfunction of the minor papilla [7]. The most relevant diagnostic marker of paraduodenal pancreatitis is the presence of cystoids in the duodenal wall that appears typically thickened. These cystoids can grow up to few centimeters in size. Duodenal wall cysts are formed from small dilated pancreatic ducts. The lumen of cysts and dilated ducts can contain calcium crystals, degenerated polymorphonuclear cells, or protein clusters [8].

All the abovementioned facts indicate that paraduodenal pancreatitis is characterized by well-defined histomorphology, clinical, and diagnostic patterns. How-
Table 1. Plasmatic IgG4 levels (higher than $135 \mathrm{mg} / \mathrm{dL}$ ) in patients with pancreatic cancer

\begin{tabular}{llll}
\hline Patient number & $\begin{array}{l}\text { Age at the time } \\
\text { of diagnosis, years }\end{array}$ & Gender & IgG4, mg/dL \\
\hline 1 & 48 & $\mathrm{M}$ & 189.0 \\
2 & 54 & $\mathrm{M}$ & 144.6 \\
3 & 58 & $\mathrm{M}$ & 153.8 \\
4 & 71 & $\mathrm{M}$ & 166.7 \\
5 & 64 & $\mathrm{M}$ & 169.9 \\
6 & 60 & $\mathrm{M}$ & 176.3 \\
7 & 70 & $\mathrm{M}$ & 188.5 \\
8 & 59 & $\mathrm{M}$ & 195.5 \\
9 & 66 & $\mathrm{M}$ & 288.6 \\
10 & 71 & $\mathrm{~F}$ & 157.3 \\
11 & 74 & $\mathrm{M}$ & 212.9 \\
12 & 60 & $\mathrm{~F}$ & 233.6 \\
13 & 68 & $\mathrm{M}$ & 179.5 \\
14 & 73 & $\mathrm{M}$ & 298.4 \\
Average & $\mathbf{6 4 . 0}$ & & 196.8 \\
\hline
\end{tabular}

IgG4, immunoglobulin G4; M, male; F, female.

ever, finding of paraduodenal pancreatitis does not allow distinguishing between the manifestation of PC or the manifestation related to autoimmune form of pancreatitis.

In this context, in terms of diagnosis of early forms of PC in particular, the relation of autoimmune form of chronic pancreatitis and PC is deemed to be of great importance. The question is how frequent is the finding of immunoglobulin G4 (IgG4) positivity specifically in patients with PC and how many patients with elevated serum IgG4 levels are affected with AIP in the simultaneous presence of PC. And finally, a focal form of pancreatopathy localized in the pancreatic head and manifested under disguise of paraduodenal pancreatitis is often dependent on histology exams in terms of establishing an etiology that leads to finding of paraduodenal pancreatitis. Currently, autoimmune form of pancreatitis is classified into 2 subtypes: type 1 referred to as lymphoplasmatic sclerosing pancreatitis and type 2 referred to as idiopathic duct centric pancreatitis.

\section{Materials and Methods}

In years 2012-2017, IgG4 serum levels were examined in 115 patients with histologically confirmed PC. The serum level above $135 \mathrm{mg} / \mathrm{dL}$ was considered as a pathologic value. By consensus, the value conclusively suggestive of AIP is double the indicated value. 

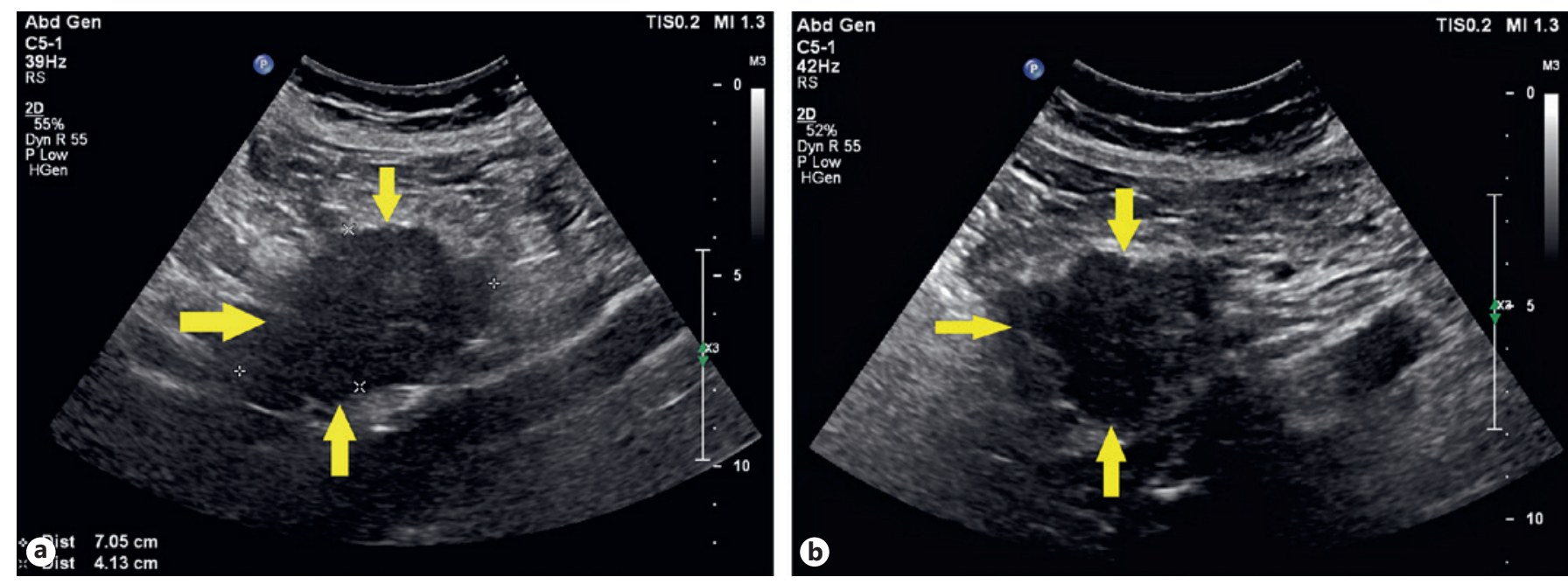

Fig. 1. Abdominal ultrasound. Sagittal slice (a), transversal slice (b): arrows show enlarged and edematous (hypoechogenic) pancreatic head without visible pancreatic duct.

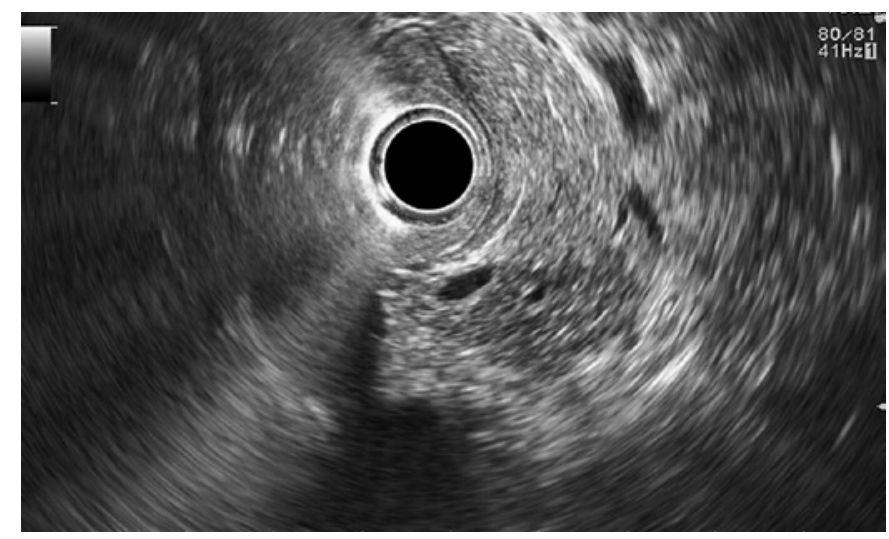

Fig. 2. Endoscopic ultrasound: oval hypoechogenic lesion in pancreatic head surrounding nondilated main pancreatic duct.

Patients with PC and elevated IgG4 level had tested their histological resection specimens or bioptic specimens from pancreatic lesion, with targeted detection of the presence of IgG4 and plasmocytes in the pancreatic tissue and changes characteristic for type 1 AIP, such as storiform fibrosis or venulitis, resp. obliterative phlebitis. The study was approved by the Ethical Committee of the University Hospital Ostrava (according to the Declaration of Helsinki).

\section{Results}

A plasmatic IgG4 level in 115 patients with diagnosed PC was higher than $135 \mathrm{mg} / \mathrm{dL}$ in 14 patients $(12.2 \%)$. Out of them, 2 patients $(1.7 \%)$ revealed a serum IgG4 level higher than double the normal value, that is, higher than $270.0 \mathrm{mg} / \mathrm{dL}$ (Table 1). The average age of patients with an elevated IgG4 level was 64.0 years. All patients with elevated IgG4 levels had histology exams of the pancreatic tissue performed in order to detect the presence of IgG4 in the tested issue as well as histological changes characteristic for AIP. In total, 2 cases out of all were histologically found to have an abundant positivity of IgG4 in the pancreatic tissue, in these 2 patients revealed a serum IgG4 level $>270.0$ $\mathrm{mg} / \mathrm{dL}$ (Table 1 patient number 9 and 14). Histological finding characteristic for AIP was found in 1 patient with PC and an elevated serum IgG4 level over twice the normal value. This patient had initially abdominal ultrasonography done and his findings were concluded as a focal lesion in the pancreatic head (Fig. 1a, b). Since his serum IgG4 level was higher than $270.0 \mathrm{mg} / \mathrm{dL}$, he was recommended to undergo endoscopic ultrasonography, which revealed paraduodenal pancreatitis in the pancreatic head region (Fig. 2). Fine needle biopsy of the pancreatic head focal lesion was performed under endoscopic ultrasonographic guidance. The finding was indicative of PC (Fig. 3). The patient was recommended to undergo surgical resection, confirming PC (Fig. 4) simultaneously with histological findings of AIP (Fig. 5a, b).

Diagnosis of early cancer changes, particularly differentiating of AIP from PC can be problematic at times. A targeted biopsy of the pancreas is the method of choice in cases suspected from a focal form of AIP and we recommend to prefer it over other modalities, such as, for example, response to steroid therapy. 


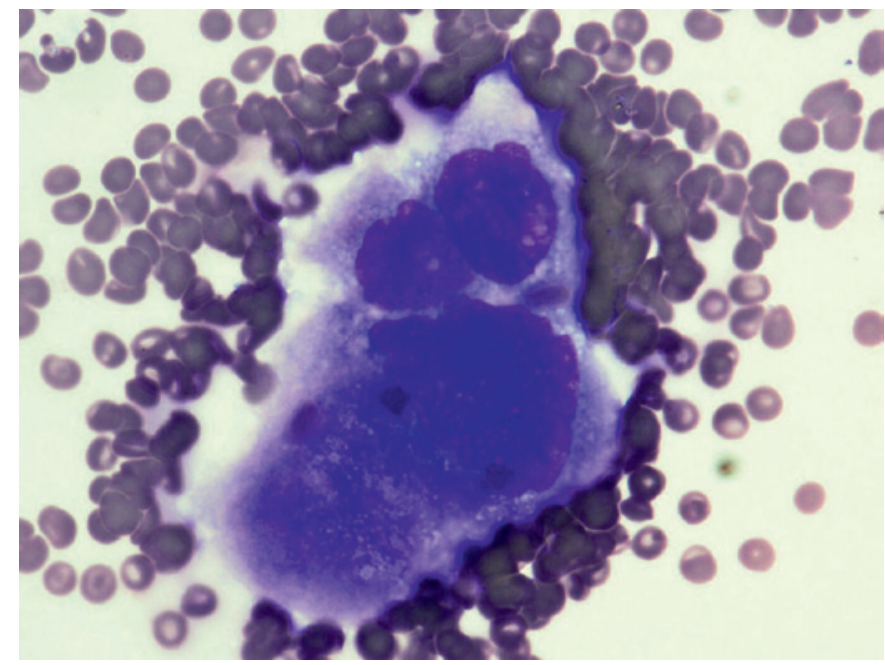

Fig. 3. Cytology - malignant cells of pancreatic adenocarcinoma, May-Grünwald Giemsa staining, 400×.

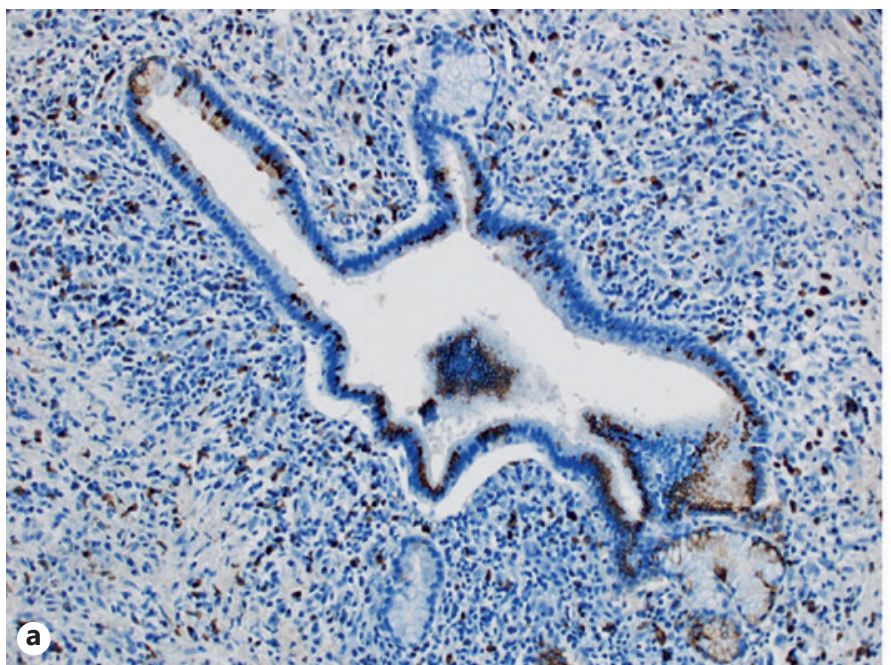

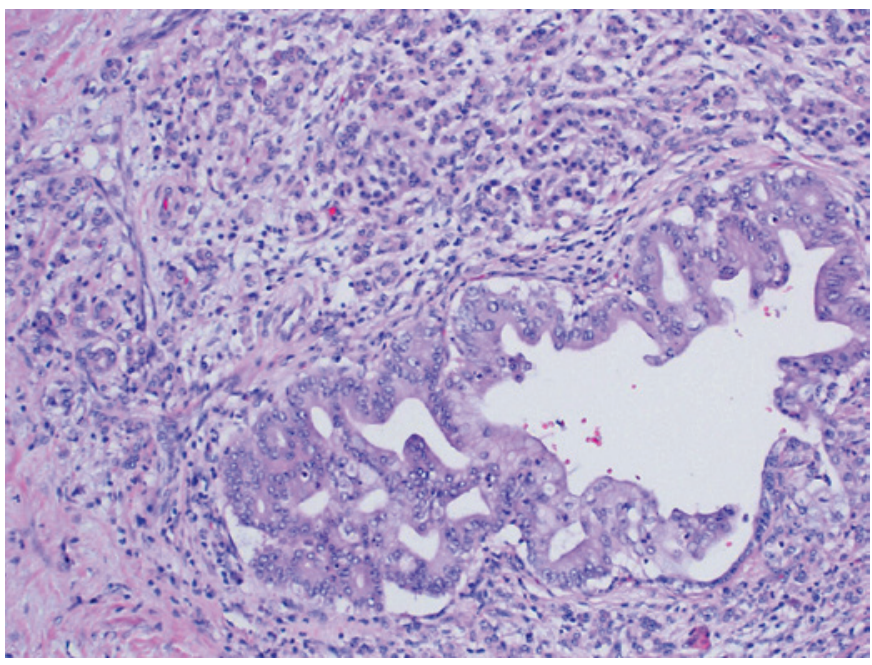

Fig. 4. Histopathology - structures of pancreatic parenchyma with diffuse lymphoplasmocellular infiltrate and a focus of a well-differentiated adenocarcinoma, HE staining, 200×.

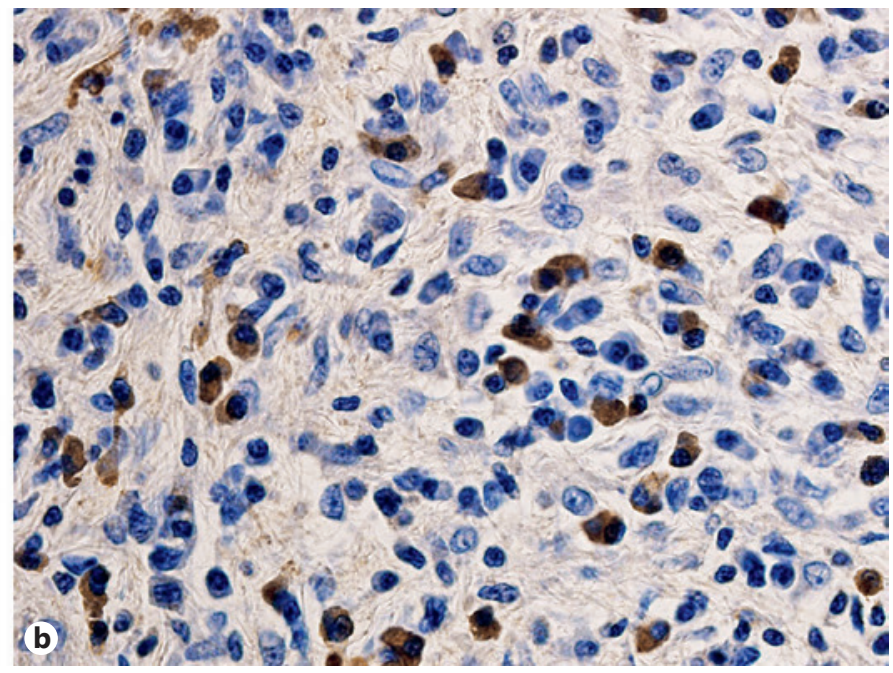

Fig. 5. a IgG4 immunohistochemical staining - mild periductal fibrosis with IgG4-positive plasmocytes in the dense round cell infiltrate, 100×. b IgG4 immunohistochemical staining - round cell lymphoplasmocytic infiltrate with IgG4-positive plasmocytes, 400×.

\section{Discussion}

In terms of differential diagnosis of PC from AIP, the current approach is based on 2 recommended models, the so called Japanese model published by Kamisawa et al. [9] and the model published by Chari et al. [10] from Rochester.

The Japanese model recommends to use IgG4 level measurement, the so-called cross-sectional pancreatic imaging and endoscopic retrograde cholangiopancreatography. Based on the Japanese model, the presence of the following 3 features on computer tomography (CT) imaging indicates the positive finding - the presence of hypoattenuating capsule rim - that is, low-density rim, delayed pancreas enhancement, and atrophic changes of the parenchyma in the pancreatic body and tail.

Endoscopic retrograde cholangiopancreatography may show slightly uneven pancreatic duct with diffuse stenoses, with no dilations observed in the course of the main pancreatic duct and stenotic changes of its secondary branches present. 
The American model prefers to use CT out of other imaging modalities, in conjunction with IgG4 level measurement and evaluation of other organ involvement (using CT imaging). If these diagnostic approaches cannot provide satisfactory results, pancreatic biopsy is recommended or a steroid trial, respectively. In case an accurate diagnose still cannot be established, surgical revision is indicated. From serology tests, CA19-9 testing is recommended and its level higher than $150 \mathrm{U} / \mathrm{mL}$ is considered as the level with a high possibility related to PC.

In 2014, a team from Taiwan published results of serum CA19-9 and IgG4 testing in differential diagnosis between PC and a focal form of AIP [11]. As study results suggested, serum IgG4 levels higher than twice the normal value combined with CA19-9 lower than $85 \mathrm{U} / \mathrm{mL}$ are important diagnostic markers that support in given values the diagnosis of focal AIP and allow for distinguishing this diagnosis from PC.

In our group of patients with histologically verified PC, in 14 patients (12.2\%) we have detected serum IgG4 levels elevated above the upper limit of normal value 135 $\mathrm{mg} / \mathrm{dL}$. An average IgG4 level in this group was $196.8 \mathrm{mg} /$ dL. Out of 14 patients with elevated IgG4, 2 patients were found with serum IgG4 elevated above twice the normal value $(270 \mathrm{mg} / \mathrm{dL})$. Twice the normal value is one of the diagnostic features suggestive of AIP.

In histological specimen with the finding of $\mathrm{PC}$ and elevated serum IgG4 levels in patients with serum IgG4 level twice the upper limit of the norm, we have found an abundant presence of IgG4 in these histological specimens. In one of these patients - according to current recommended diagnostic criteria [12] - a histomorphological finding was concluded as AIP, that is, this patient has PC in the simultaneous presence of AIP.

It remains unclear why patients with $\mathrm{PC}$ are found to have elevated IgG4 levels without the presence of AIP. IgG4 is a remarkable antibody in terms of both structure and functions. In healthy individuals, the IgG4 molecule accounts for $5.0 \%$ of all IgG spectrum. IgG4 positivity is associated with a full range of conditions, mainly with a group of diseases known as IgG4-related diseases. The most common condition out of this group is AIP, often associated with multiple organ involvement, such as biliary cholangitis, Mikulicz syndrome, IgG4-positive pneumonitis, retroperitoneal fibrosis, lymphadenopathy, and IgG4-positive mastitis. IgG4 elevation is found in many allergic conditions, IgG4-positive thyroiditis, pericarditis, inflammatory pseudotumors, mediastinal fibrosis, as well as other conditions $[13,14]$.

Physiologically, an IgG4 response is induced by long term or repeated antigen exposure [15]. Similarly as with $\mathrm{IgE}, \mathrm{IgG} 4$ production is primarily regulated by Th2 $\mathrm{cy}-$ tokines, such as interleukin 4 and interleukin 13 [16]. It has not been satisfactory explained so far, what is the role of IgG4 in patients with PC or whether it has a solely diagnostic value or it possibly reflects autoimmune processes in the presence of PC. There are 2 possible explanations for an abundant elevation of IgG4. The first is that antibodies may behave as tissue destructive immunoglobulins. The second is that there may be an overt expression of these antibodies, as a result of a response to an unknown primary inflammatory stimulus. However, these theories do not provide a definite explanation of the relations of IgG4 to PC, although an existence of AIP in the simultaneous presence of PC cannot be ruled out. Further research is needed to address this issue.

\section{Statement of Ethics}

Patients have given their written informed consent, and the study protocol has been approved by the by the Ethical Committee of the University Hospital Ostrava (according to the Declaration of Helsinki).

\section{Disclosure Statement}

All authors declare no conflicts of interest in relation to this article.

\section{Grants or Financial Support}

No grants or financial support were provided.

\section{References}

1 Zamboni G, Capelli P, Scarpa A, Bogina G, Pesci A, Brunello E, et al. Nonneoplastic mimickers of pancreatic neoplasms. Arch Pathol Lab Med. 2009 Mar;133(3):439-53.

2 Adsay NV, Zamboni G. Paraduodenal pancreatitis: a clinico-pathologically distinct en- tity unifying "cystic dystrophy of heterotopic pancreas", "para-duodenal wall cyst", and "groove pancreatitis". Semin Diagn Pathol. 2004 Nov;21(4):247-54.

3 Stolte M, Weiss W, Volkholz H, Rösch W. A special form of segmental pancreatitis: "groove pancreatitis". Hepatogastroenterology. 1982 Oct;29(5):198-208

4 Potet F, Duclert N. [Cystic dystrophy on aberrant pancreas of the duodenal wall]. Arch Fr Mal App Dig. 1970 Apr-Mar;59(4):22338. 
5 Fléjou JF. Paraduodenal pancreatitis: a new unifying term and its morphological characteristics. Diagn Histopathol. 2012;18(1):31-6.

6 Arora A, Dev A, Mukund A, Patidar Y, Bhatia V, Sarin SK. Paraduodenal pancreatitis. Clin Radiol. 2014 Mar;69(3):299-306.

7 Stevens T, Conwell DL, Zuccaro G. Pathogenesis of chronic pancreatitis: an evidencebased review of past theories and recent developments. Am J Gastroenterol. 2004 Nov; 99(11):2256-70.

8 Fléjou JF, Potet F, Molas G, Bernades P, Amouyal P, Fékété F. Cystic dystrophy of the gastric and duodenal wall developing in heterotopic pancreas: an unrecognised entity. Gut. 1993 Mar;34(3):343-7.

9 Kamisawa T, Imai M, Yui Chen P, Tu Y, Egawa N, Tsuruta K, et al. Strategy for differenti- ating autoimmune pancreatitis from pancreatic cancer. Pancreas. 2008 Oct;37(3):e62-7.

10 Chari ST, Takahashi N, Levy MJ, Smyrk TC, Clain JE, Pearson RK, et al. A diagnostic strategy to distinguish autoimmune pancreatitis from pancreatic cancer. Clin Gastroenterol Hepatol. 2009 Oct;7(10):1097-103.

11 Chang MC, Liang PC, Jan S, Yang CY, Tien YW, Wei SC, et al. Increase diagnostic accuracy in differentiating focal type autoimmune pancreatitis from pancreatic cancer with combined serum IgG4 and CA19-9 levels. Pancreatology. 2014 Sep-Oct;14(5):366-72.

12 Shimosegawa T, Chari ST, Frulloni L, Kamisawa T, Kawa S, Mino-Kenudson M, et al.; International Association of Pancreatology. International consensus diagnostic criteria for autoimmune pancreatitis: guidelines of the
International Association of Pancreatology. Pancreas. 2011 Apr;40(3):352-8.

13 Stone JH, Zen Y, Deshpande V. IgG4-related disease. N Engl J Med. 2012 Feb;366(6):539_ 51.

14 Islam AD, Selmi C, Datta-Mitra A, Sonu R, Chen M, Gershwin ME, et al. The changing faces of IgG4-related disease: clinical manifestations and pathogenesis. Autoimmun Rev. 2015 Oct;14(10):914-22.

15 Aalberse RC, Stapel SO, Schuurman J, Rispens T. Immunoglobulin G4: an odd antibody. Clin Exp Allergy. 2009 Apr;39(4):46977.

16 Nirula A, Glaser SM, Kalled SL, Taylor FR. What is IgG4? A review of the biology of a unique immunoglobulin subtype. Curr Opin Rheumatol. 2011 Jan;23(1):119-24. 